\title{
DIE KUNST DES INTAVOLIERENS: GEBUNDENHEIT UND FREIHEIT
}

Johannes RiNG

\begin{abstract}
A big part of $16^{\text {th }}$ century keyboard repertory was intabulated vocal music; arrangements of motets, sets of masses and chansons. Marcant and sometimes little known examples show the different functions of intabulations: repertoire for the organists (liturgical function), music of delight and pedagogical functions. As a part of Ars Organistum intabulations were the basis of professional musical studies, learning to improvise organ music and to compose music. Masters like $\mathrm{H}$. Scheidemann, the both Gabrielis, J. de Lublin, P. Philips, the both Cabezóns, some english and french anonym composers demonstrate that intabulations are much more than keyboard reductions - artful arrangements with their own life and character on the way to artful paraphrases. The intentions of the composers / arrangers in connection with the possibilities of the keyboard instruments definite the character and the step of transforming a vocal set in a (new) piece of keyboard music.
\end{abstract}

\section{Zusammenfassung}

Ein großer Teil des Musikrepertoires für Tasteninstrumente im 16. Jahrhundert bestand aus intavolierter Vokalmusik - Bearbeitungen von Motetten, Messsätzen und Chansons. Markante und teilweise wenig bekannte Beispiele zeigen verschiedene Fünktionen der Intavolierungen. Zu nennen sind das Organistenrepertoire (liturgisches Umfeld), die Musik zur Erbauung und Unterhaltung und die pädagogische Funktion. Als Bestandteil der Ars Organistum waren Intavolierungen wichtig um professionell das Improvisieren von Musik und das Komponieren zu erlernen. Exemplarisch dokumentieren besonders H. Scheidemann, die beiden Gabrielis, J. de Lublin, P. Philips, die beiden Cabezóns und einige anonyme Komponisten aus England und Frankreich, dass ihre Kunst des Intavolierens weit über den Übertragungsmodus hinausgeht, bis hin zum virtuosen Arrangement und zur kunstvollen Paraphrase. Die Intentionen der Komponisten in Verbindung mit den Möglichkeiten der Tasteninstrumente prägen den Charakter und den Umformungsgrad einer Intavolierung.

\section{Präludium}

Es ist gut vorstellbar, wie Heinrich Scheidemann einem interessierten hanseatischen Publikum mit der kunstvollen Bearbeitung bekannter Motetten prominenter Komponisten seine musikalische Kompetenz unter Beweis stellte und die klanglichen Möglichkeiten der großen Orgel der Hamburger Hauptkirche St. Katherinen vorführte. Ähnlich muss der Engländer Peter Philips in Antwerpen die wohlhabende Bürgerschaft am Virginal mit der virtuosen Bearbeitung populärer italienischer Madrigale beeindruckt haben.

Als Scheidemann und Philips in der ersten Hälfte des 17. Jahrhunderts ihre kunstvollen Intabulierungen erklingen ließen, schaute die Praxis des Intabulierens von ihrem letzten Höhepunkt auf eine dreihundertjährige Geschichte zurück.

Anuario Musical, 58 (2003) 
Beginnend mit dem frühen 14. Jahrhundert bis in das 17. Jahrhundert hinein entstand ein umfangreiches, schriftlich aufgezeichnetes Instrumentalrepertoire mit einem hohen Anteil abgesetzter Stücke (= Intabulierungen / Intavolierungen). ${ }^{1}$ Hauptsächlich wurden Motetten, Madrigale, Chansons und Messsätze für Tasteninstrumente und Saiteninstrumente bearbeitet. Im Brennpunkt folgender Ausführungen stehen Intabulierungen für Orgel und andere Tasteninstrumente.

Durch ihre ausgeprägte tastenmusikalische Idiomatik haben viele Intabulierungen gegenüber ihren Vorlagen den Rang von eigenständigen Stücken. ${ }^{2}$ Unter Berücksichtigung der spielpraktischen Gegebenheiten der Tasteninstrumente sollen verschiedene Beispiele zeigen, dass die Spannbreite der Bearbeitungen von wörtlicher Umsetzung des Notentextes über einen ausgearbeiteten und reich verzierten Instrumentalsatz ${ }^{3}$ bis hin zur freien Paraphrasierung einer Vorlage reichen.

Wir können fünf Intabulierungstypen unterscheiden: ${ }^{4}$

- Der 1. Typus: Die Stimmlagen sind gut nachvollziehbar. Die Stimmen verteilen sich gleichmäßig auf beide Hände. Koloraturen können bei gleichzeitig ausgehaltenen Noten oder Akkorden ausgeführt werden.

- Beim 2. Typus vernachlässigt die griffmäßige Umsetzung der Vorlage die Stimmführung. Lange Notenwerte werden durch Repetition rhythmisiert und belebt. Die obere Stimme dominiert beim Kolorieren.

- Der 3. Typus nährt sich aus Eigenarten des säkularen Tasteninstrumentenstils. Über parallel geführten Grundakkorden bewegt sich im Diskant ein überwiegend gleichförmiges, virtuoses Figurenwerk.

- Als 4. Typus möchte ich die quasi unbearbeitete Umsetzung der vokalen Vorlage in ein Tastenarrangement nennen.

- Einen 5. Typus, der die Klangfarben betrifft, können wir im Zusammenhang mit Heinrich Scheidemanns Motettenbearbeitungen für die norddeutsche Orgel kennen lernen.

1. Intavolieren, intabulieren und absetzen (sowie ihre Substantive) werden synonym verwendet. Als knapper Überblick über die Intabulierungen im 14. und 15. Jahrhundert und das Buxheimer Orgelbuch (1470) liest sich gut: Reinhard Schäfertöns, Chansons für Orgel, Motetten auf der Laute. Intabulierung als Bearbeitung, in: Musikalische Metamorphosen, Formen und Geschichte der Bearbeitung, hrsg. von Silke Leopold [Bärenreiter Studienbücher Musik, Bd. 2]. S. 11-20.

2. Arnfried Edler, Gattungen der Musik für Tasteninstrumente. Teil 1: Von den Anfängen bis 1750. In: Handbuch der musikalischen Gattungen, Bd. 7, hrsg. von Siegfried Mauser, Laaber 1997. S. 18 [= Edler, 1997].

3. Howard Mayer Brown, Art. Intabulation, in: NGroveD, Vol. 9. S. 256 und Edler, 1997. S. 18. Edler rollt hier kurz die Geschichte dieser Gattungsgruppe auf.

4. Zum Typus 1 bis 3 siehe Harald Vogel, Zur instrumentalen Aufführungsweise des Motettenrepertoires unter besonderer Berücksichtigung der Orgelintabulierungen, in: Orgel und Orgelspiel im 16. Jahrhundert. Tagungsbericht, Innsbruck 9.-12.6.1977, hrsg. von Walter Salmen, Innsbruck 1978. [= Orgel im 16. Jahrhundert] S. 98ff. [= Vogel, 1978]. 
In der zweiten Hälfte des 16. Jahrhunderts thematisierten einige Musiktheoretiker wie Vincenzo Galilei und Lodovico Zacconi ${ }^{5}$ in ihren Traktaten das Intabulieren. Zuvor beschrieb um 1520 Hans Buchner in seinem Fundamentum ${ }^{6}$ drei Elemente, welche die Organistenkunst (Ars Organistum) prägen: 1. Via ludendi (Beherrschung der Spieltechnik), 2. Ratio transferendi compositas cantiones in forma organistarum, quam tabulaturam vocant (Absetzen mehrstimmiger vokaler Stücke für die Orgel) und 3. Ratio quemvis cantum planum redigenti ad iustas duarum, trium aut plurium vocum diversarum symphonias (Kontrapunktlehre = die Cantus-firmus-gebundene mehrstimmige Satzkunst, z.B. Versetten für die Alternatimpraxis). ${ }^{7}$ Dieses elementare Dreigestirn der Fundamentpraxis versetzt Organisten in die Lage zu arrangieren und improvisieren. ${ }^{8}$

35 Jahre nach Buchner nennt der spanische Komponist und Theoretiker Padre Fray Juan Bermudo im 41. Kapitel seines Traktates Libro llamado declaración de instrumentos musicales gleichfalls das Absetzen vokaler Vorlagen als Kriterium für einen guten Instrumentalmusiker. ${ }^{9}$ Bermudo nennt drei Arten des Absetzens. Die erste verlangt einen geübten Musiker, der polyphone Musik aus dem Chorbuch absetzen kann. Die zweite Art ist für weniger geübte Spieler bestimmt. Als Hilfe soll der Musiker die Mensuralgesänge mit Ordnungsstrichen versehen, so dass die Aufteilung dem Spieler eine leichtere Orientierung verschafft. Die dritte Art ist für ungeübte Musiker. Diese sollen eine Zeichentabulatur herstellen. Die Intabulierungsübungen finden zunächst auf dem häuslichen Monachordio statt. Es ist für alle Tastenmusiker, ob Organisten oder Cembalisten, gleichermaßen das Übungsinstrument der Wahl.

Weiter gibt Bermudo den Hinweis, dass man sich, vorausgesetzt man ist ein technisch versierter Spieler („En teniendo buenas manos“), mit qualitativ guter Musik befassen soll. Bermudo zielt hier wohl auf musiktheoretische Kenntnisse ab, die man sich durch Intabulieren guter Musik aneignen kann. Ein erstes Intabulieren kann man mit den einfachen, musikalisch aber wertvollen villancicos von Juan Vasquez üben. „Despues poned musica de Iosquin, de Adriano, de Iachet mantuano, del maestro Figueroa, de Morales, de Gombert, y de algunos otros

5. Galilei: Fronimo: dialogo ..., et necessarie regole del intavolare ..., Venedig, 1568/69 und Zacconi: Prattica di musica utile et necessaria si al compositore ..., Venedig, 1592. Siehe Frieder Rempp: Elementar-und Satzlehre von Tinctoris bis Zarlino, in: Geschichte der Musiktheorie Band 7: Italienische Musiktheorie im 16. und 17. Jh., Darmstadt, 1989.

6. Fundamentum, sive ratio vera, quae docet quemvis cantum planum ..., ed. in: Carl Paesler, Das Fundamentbuch von Hans von Constanz, in: Vierteljahresschrift für Musikwissenschaft, Band V (1889). S. 1-192.

7. Luigi Ferdinando Tagliavini, Orgel und Orgelmusik, in: Geschichte der katholischen Kirchenmusik, hrsg. von Karl Gustav Fellerer, Bd. I. Von den Anfängen bis zum Tridentinum, Kassel, 1972. S. 465.

8. Arnfried Edler, Der nordelbische Organist. Studien zu Sozialstatus, Funktion und kompositorischer Produktion eines Musikberufes von der Reformation bis zum 20. Jahrhundert. (= Kieler Schriften zur Musikwissenschaft, Band XXIII) Kassel u.a., 1982. S. 151. [= Edler, 1982].

9. Vergl. Otto Kinkeldey, Orgel und Klavier in der Musik des 16. Jahrhunderts, Leipzig, 1910, Neudruck Wiesbaden, 1984. S. 20. [= Kinkeldey]. Bermudos Libro llamado declaración ist ediert in: Fray Juan Bermudo, Declaración de Instrumentos musicales, Osuna, 1555, hrsg. von Macario Santiago Kastner, Documenta Musicologica, Kassel u.a., 1957. Faksimile. [= Declaración]. 
semejantes. “10 Bermudo nennt hier einige der bedeutendsten frankoflämischen, italienischen und spanischen Komponisten des 15. und 16. Jahrhunderts.

\section{III.}

Der hohe Anteil der Intavolierungen im Tastenrepertoire ist auf das Parodiephänomen im 16. Jahrhundert zurückzuführen. „Reizvoller als die Erfindung einer neuen musikalischen Substanz fand man offenbar vielfach den Aufbau einer Komposition auf der Substanz einer bereits vorhandenen, ganz besonders dann, wenn Gattungsgrenzen überschritten wurden. “11

Wie wir bei Bermudo erfahren können, waren professionelle Musiker in der Lage, aus dem Stehgreif heraus, beispielsweise aus dem Chorbuch abzusetzen. Viele Komponisten des 16. und der ersten Hälfte des 17. Jahrhunderts stellten diese Fähigkeit öffentlich auf den Prüfstand. ${ }^{12}$ Dagegen weist der hohe Anteil handschriftlich und gedruckt überlieferter Intabulierungen auf pädagogische Intentionen hin. Orgelbearbeitungen vokaler Vorlagen dienten als Lehrmittel. Michael Praetorius schreibt im Vorwort zu seinen Musae Sioniae von 1609: „So hat vors ander der Autor auff etlicher Organisten instendiges anhalten Vier deutscher Psalmen ohne Text in diesem Siebenden Theile hinten an drucken lassen damit ein angehender Organist welchem sie etwa gefallen moechten dieselben also zum gebrauch aus den Noten wiederuemb in die Tabulatur bringen koenne." ${ }^{13}$

Doch zielt der Vorschlag von Praetorius sicher nicht auf das mechanische Absetzen einer vokalen mehrstimmigen Vorlage in ein Clavierstück. Als eigentliche Intention können wir annehmen, dass sich ein Orgel- bzw. Musikstudent durch das Absetzen bewährter vokaler Vorlagen mit der Technik des Komponierens vertraut machen sollte.

Gegen Ende des 16. Jahrhunderts erweiterten viele Organisten die praxisorientierten spieltechnischen Elemente durch das Komponieren. Das kontrapunktische Improvisieren der Fundamentpraxis Buchners (s.o.) stellt das Bindeglied zwischen handwerklicher Spielpraxis und der theoretisch fundierten Vokalmusik dar. Anders formuliert: die Organisten verbanden Formen der Vokalmusik mit instrumentaler Idiomatik. ${ }^{14}$

Neben Praetorius' pädagogischer Intention spielt die gottesdienstliche Verwendung dieses Tastenrepertoires eine große Rolle. Dieses kam zur Aufführung, wenn der mehrstimmige Chor

10. Declaración, folio 60 des libro quarto. "Danach setze die Musik von Iosquin, Adriano, Iachet mantuano, des maestro Figueroa, von de Morales und Gombert ab."

11. Edler, 1997. S. 18. Siehe auch Hans Klotz, Über die Orgelkunst der Gotik, der Renaissance und des Barock, Kassel u.a., 1986. S. 7 u. 8. [= Klotz].

12. Willi Apel, Neapolitan Links between Cabezon und Frescobaldi, in: The Musical Quarterly, Vol. XXIV (1938). S. 432. [= Apel].

13. Zitiert aus Michael Praetorius, Sämtliche Orgelwerke, hrsg. von Klaus Beckmann, Wiesbaden, 1990. S. 3 \& 61. Die Erläuterungen des Komponisten haben die gleiche Relevanz für die, ebenfalls in Stimmbüchern veröffentlichten Orgelhymnen in den Hymnodia Sionia von 1611.

14. „Der Prozess der allmählichen Aneignung des Kompositionsbereiches durch die Organisten dauerte bis in die zweite Hälfte des 17. Jahrhunderts hinein, ... ." Edler, 1982. S. 156. 
abwesend war. ${ }^{15}$ Aufgabe solcher Orgelstücke war es, die Sammlung der Gottesdienstbesucher zu fördern. Orgelstücke verschiedener Faktur konnten Rahmen und Bestandteil eines Gottesdienstes sein.

Für die drei Orgelmessen der Fiori Musicali ${ }^{16}$ komponierte Girolamo Frescobaldi drei Ricercari dopo il Credo bzw. post il Credo. Diese Musikstücke wurden nach dem choralgesungenen Credo zur kontemplativen Bekräftigung der zentralen christlichen Glaubensaussage vorgetragen. Anstelle der Credo-Ricercari ist der Vortrag einer intavolierten Motette denkbar.

IV.

Solche Motetten kennen wir vom Organisten des Markusdomes in Venedig Giovanni Gabrieli. Seine sechsstimmige Motette „Exaudi Domine“ aus den Sacrae symnphoniae von 1597 lässt sich exemplarisch mit einer wahrscheinlich vom Komponisten selbst angefertigten Orgelintavolierung vergleichen. ${ }^{17}$ Wir wählen eine Neuausgabe der Motette, der eine keyboard reduction beigegeben ist, wie wir sie aus vielen Praktikerausgaben for rehearsal only kennen. Ein solcher Klavierauszug zu Probezwecken entspricht einem ersten einfachen, quasi unveränderten Intavolierungsgrad. Der Notentext der Motetten ist rhythmisch vereinfacht. Die lineare Stimmführung ist der tastenmäßigen Grifftechnik untergeordnet. Mit dieser direkten Umsetzung kann sich der interessierte Musiker einen ersten Eindruck von der Komposition verschaffen. Pettis Stimmensubstrat nimmt dem Interessenten die Arbeit des Partitur- bzw. Stimmbuchspielens ab. Dagegen empfiehlt der im Petersdom zu Rom wirkende Girolamo Frescobaldi jedem Tastenmusiker, sich auch dieser Arbeit zu widmen. "Ich erachte es von großer Wichtigkeit für die Spieler, sich im Partiturspiel zu üben, nicht nur weil ich es als notwendig betrachte für diejenigen, die sich in die Art dieser Kompositionen vertiefen wollen, sondern auch darum, dass es als Prüfstein die echten Künstler von den Unwissenden unterscheidet. "18

G. Gabrielis Motettenintavolierung zeigt einen gewissen Umformungsgrad. Ein erster augenfälliger Unterschied zwischen der vokalen Urfassung und ihrer instrumentalen Bearbeitung besteht in der Transposition. Die Intavolierung steht ein Quarte tiefer. Spieltechnische Gründe scheinen für diese Transposition nicht maßgeblich gewesen zu sein. Große Griffe, bzw. bestimmte Griffkombinationen, die durch eine verkürzte Oktav möglich wären, finden sich in dieser Bearbeitung nicht. Das Tastenstück ist ohne Orgelpedảe realisierbar. Die Transposition

15. Lydia Schierning, Die Überlieferung der deutschen Orgel- und Klaviermusik aus der 1. Hälfte des 17. Jahrhunderts, Kassel, 1961. S. 101.

16. Girolamo Frescobaldi, Orgel- und Klavierwerke, Band V, Fiori musicali 1635, hrsg. von Pierre Pidoux, Kassel u.a., 1959. [= Pidoux, 1959].

17. Die Motette ist ediert in: The Italian and Spanish Schools for 6 voices, Chester Music, Anthony G. Petti (ed.), London, 1982. S. 2-8. Die Intavolierung ist ediert in G. Gabrieli, Composizioni Per Organo Vol. III, hrsg. von Sandro Dalla Libera, Mailand, 1959/74. Zur Quellenlage siehe die Introduzione.

18. Pidoux, 1959. S. 3. 
lässt Rückschlüsse auf eine aufführungspraktische Gepflogenheit zu, bei der die Motette tiefer als notiert erklang, in diesem Fall als Quarttransposition. Außerdem ist der Stimmton der Orgel $\mathrm{zu}$ berücksichtigen, der oft über dem heutigen Kammerton lag. ${ }^{19}$

Während Pettis keyboard reduction auf rhythmische Vereinfachung abzielt, belebt Gabrieli den Orgelsatz mit rhythmischen und dynamisch-artikulatorischen Elementen. Dadurch vermeidet er klanglich unergiebige Tonwiederholungen, wie sie dem sechsstimmigen Motettensatz inhärent sind. ${ }^{20}$ Ein Notenbeispiel beleuchtet Gabrielis Vorgehensweise.

\section{N.B. 1, Motettenexposition und Anfang der Bearbeitung: ${ }^{21}$}

a) Motette:

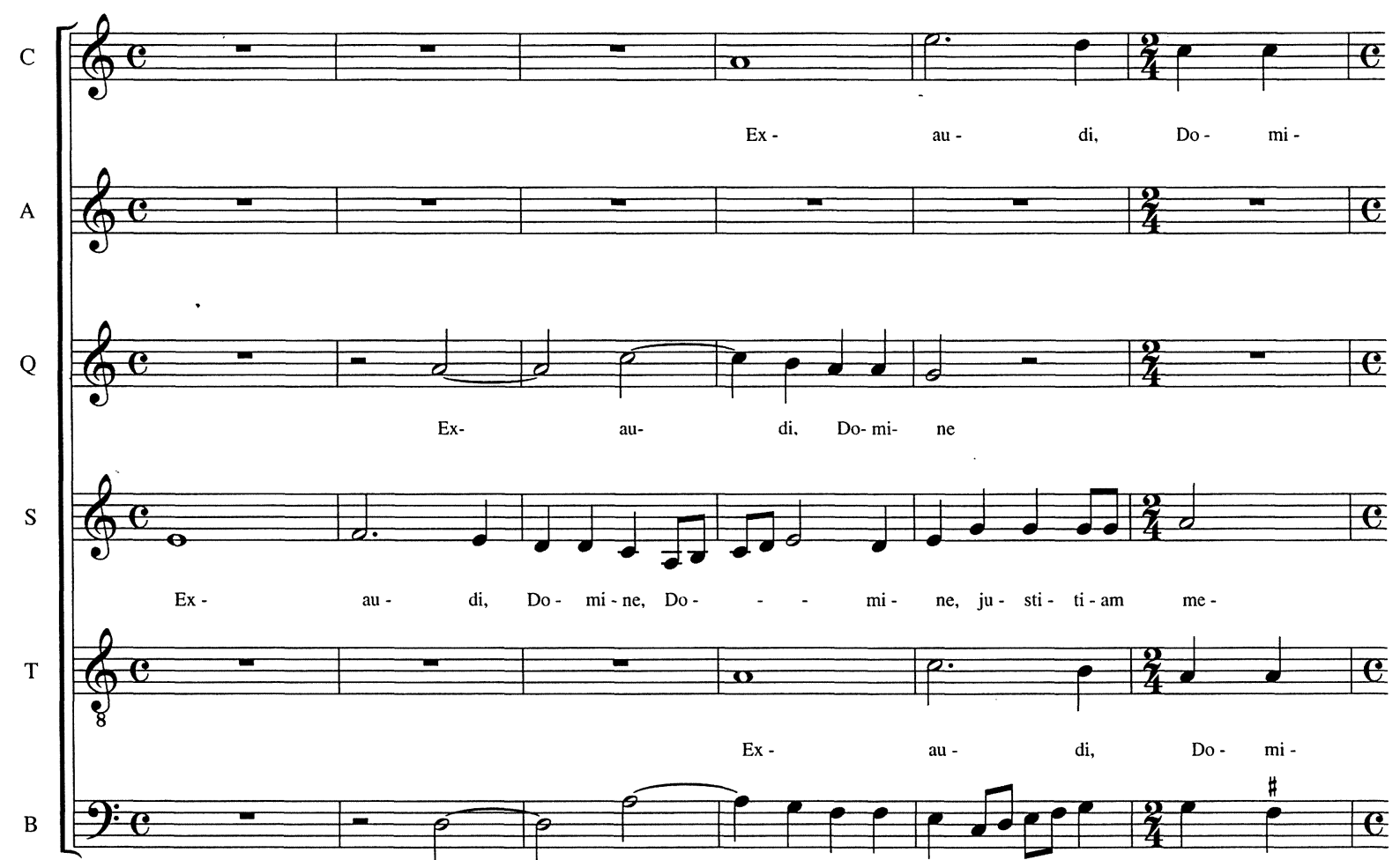

Ex -

$\mathrm{au}-$

di, Do - mi - ne, Do -

$\mathrm{mi}$ -

19. Vergl. Otto Kinkeldey, Orgel und Klavier in der Musik des 16. Jahrhunderts, Leipzig, 1910, Neudruck Wiesbaden, 1984. S. 130ff. [= Kinkeldey].

20. Vergl. hierzu Vogel, 1978. S. $94 \mathrm{ff}$.

21. Nach Petti, S. 2 und Dalla Libera, S. 1. 


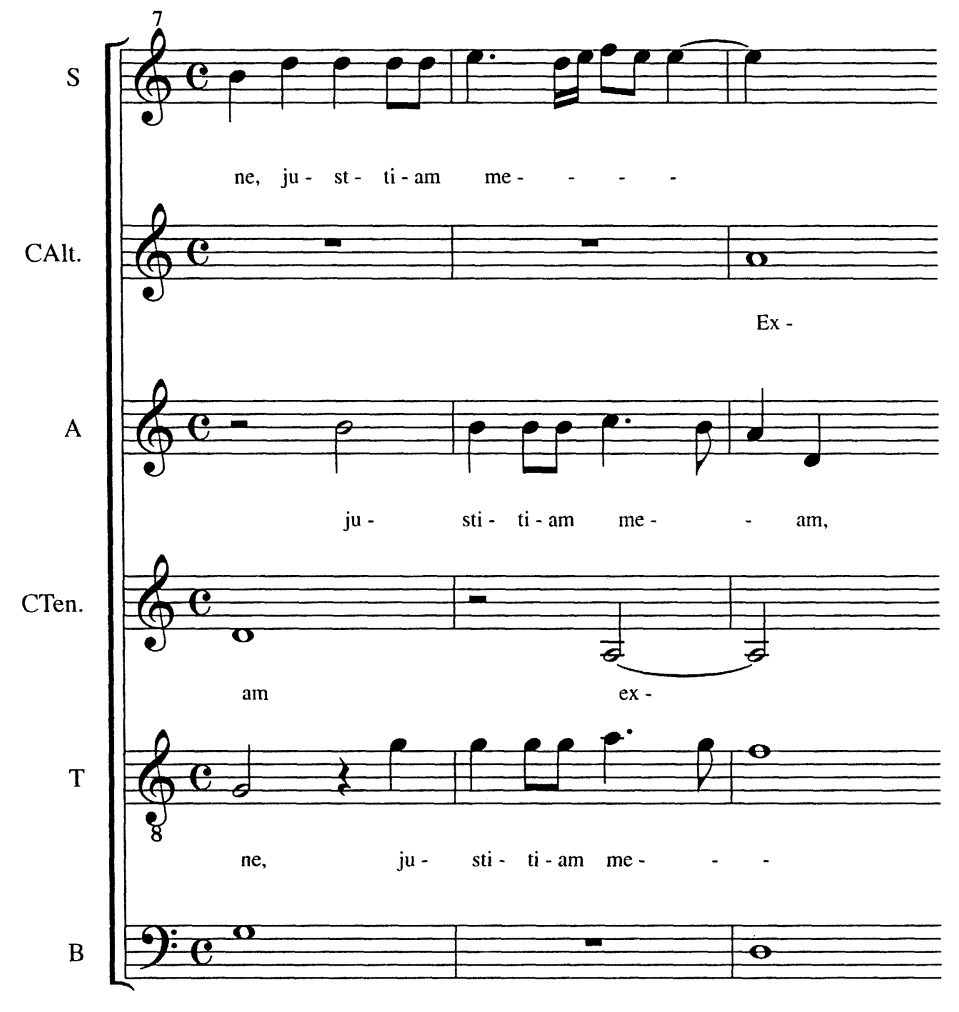

ne

ex -

b) Intavolierung:
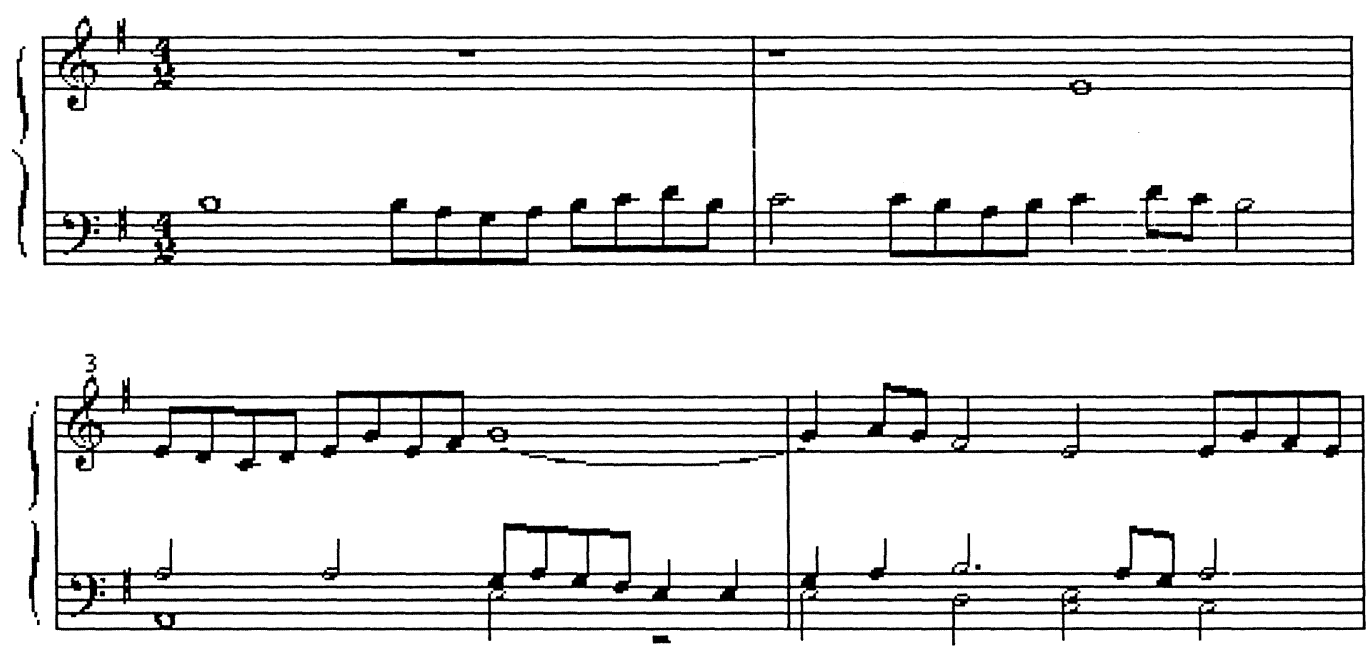


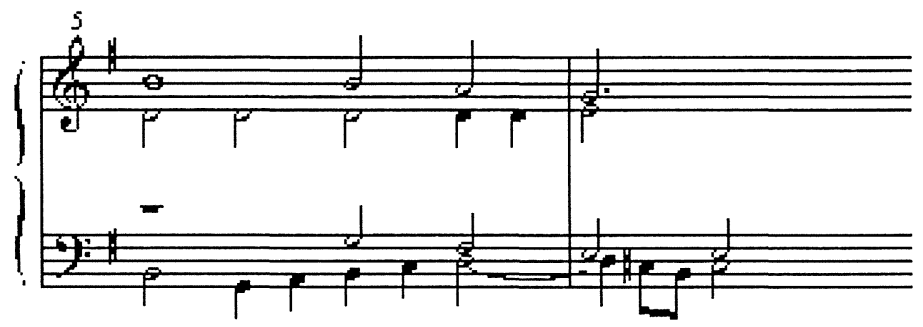

Systematisch eingesetzte kleine Achtelkoloraturen der Intavolierung lockern als Umspielungen lange Notenwerte der ruhigen Motettenexposition „Exaudi, Domine,“ auf. Sie bilden den Motor der instrumentalen Exposition. Im weiteren Verlauf der Orgelfassung wechseln kurze Passagen mit und ohne Spielfiguren ab. Dabei reagiert Gabrieli auf die Textstruktur der Motette. Der erste Wechsel tritt ein, wenn in der Exposition bei ,justitiam meam“ Vollstimmigkeit erreicht ist. Lediglich kleine Durchgänge lockern die Klangfolgen auf. Der Bassus wird am wenigsten umgeformt. Die meisten Koloraturen treten im jeweils oberen Bereich der beiden Hände bzw. Griffe auf.

Koloraturen und Durchgänge beleben nicht nur den Instrumentalsatz, sie dienen auch der musikalischen Textdarstellung. Virtuose auf- und abwärtseilende Skalen setzen den eindringlichen Ruf ,intende, intende“ des Cantus musikalisch in Szene. Dies ist ein schönes Beispiel dafür, wie Instrumentalmusik zur Darstellung des Textes der Motettenvorlage eingesetzt wird. Gabrielis Stück zeigt, dass sich ein gesteigerter musikalischer Ausdruck, der sich hier auf eine Textstelle bezieht, nicht erst bei Frescobaldi findet. ${ }^{22}$

22. Auf den Umstand der instrumentalen Textdarstellung bei Frescobaldi weist Friedrich W. Riedel in seinem Aufsatz: Manier und Manierismus in der Musik für Tasteninstrumente um 1600. Über die Anfänge des instrumentalen Stilus Fantasticus, in: Orgel im 16. Jahrhundert, S. 117. [= Riedel] hin. Musikalische Textdarstellung in Tastenmusik beschäftigt weiterhin die Forschung. Siehe z.B. Matthias Schneider, Buxtehudes Choralfantasien. Textdeutung oder „phantastischer Stil“, Kassel, 1997. Hier besonders Kapitel 1. 
N.B. 2, „Intende“ $:^{23}$

a) Motette:

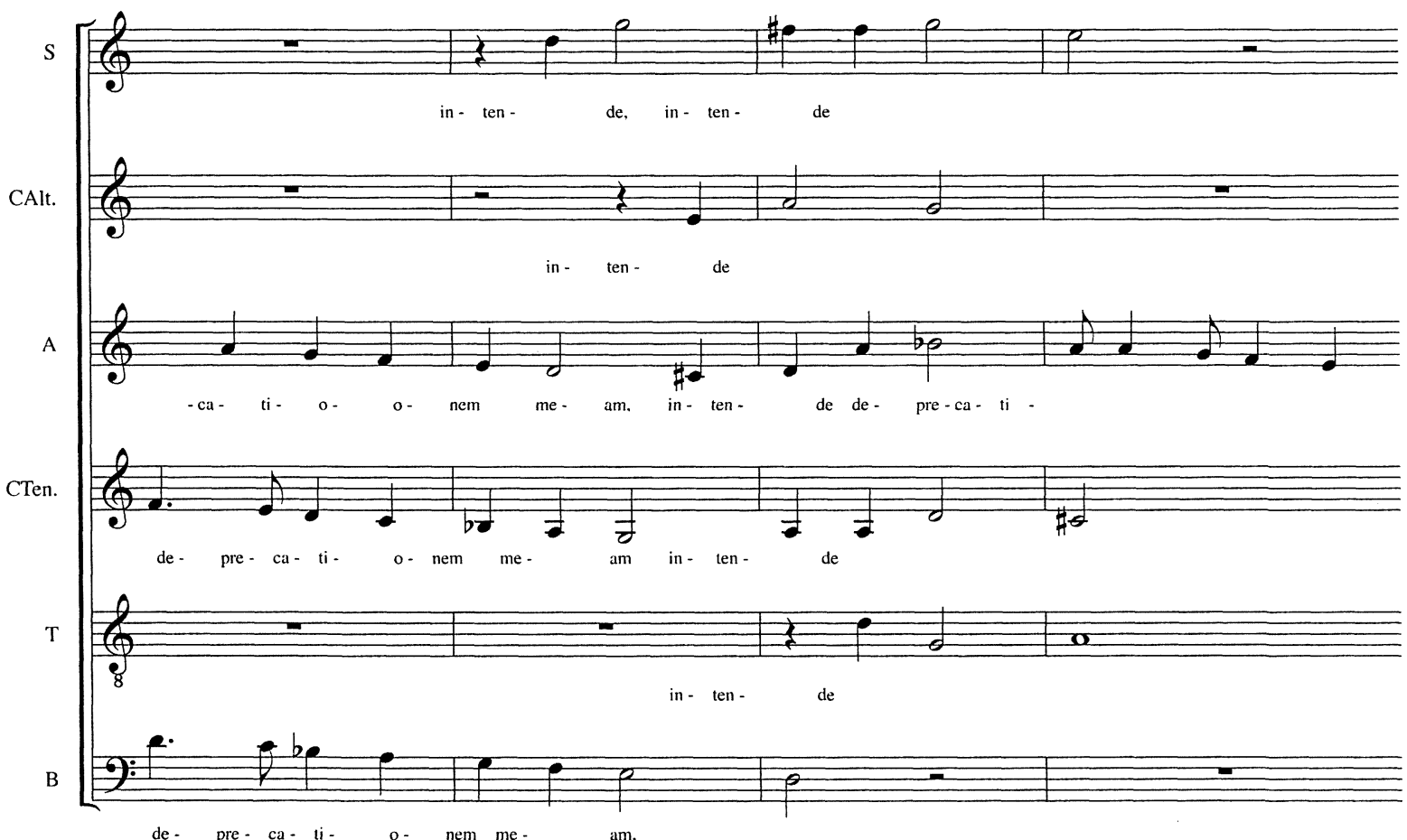

b) Intavolierung:

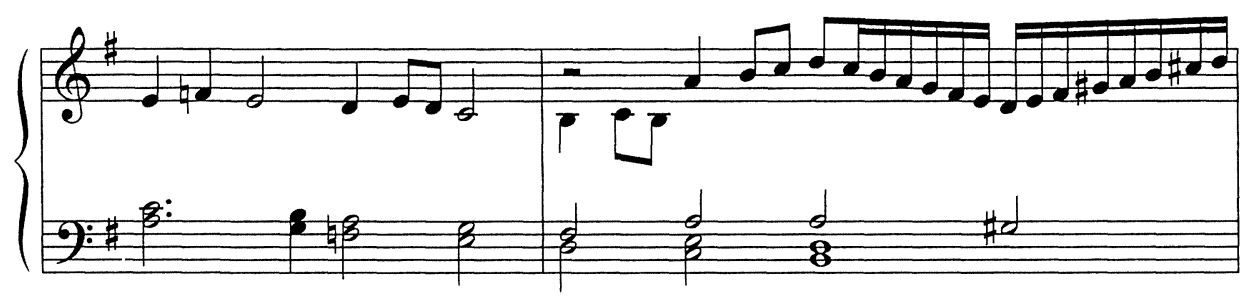

Im weiteren Verlauf der Motette dünnen Pausen den Satz aus. Die Intavolierung vollzieht die dynamische Reduktion mit kleineren Griffen nach.

23. Nach Petti, S. 4 und Dalla Libera, S. 2. 
Nach der dynamischen Ausdünnung erhält die Motette bei ,auribus percipe orationem meam" neue wortausdeutende Impulse. Kleinere Notenwerte und eine mitunter virtuose madrigalhafte Stimmenführung verdichten den Motettensatz. In der Intavolierung zeichnet der Arrangeur die dichte Folge der Klänge vollgriffig nach.

Bei „non in labiis dolosis“ wechseln die Mensur und die Satzart in der Motette. Den Wechsel in die perfekte Mensur vollzieht die Tastenbearbeitung nicht nach. Auf den homorhythmischen Satz reagiert die Intavolierung mit einer ruhigen Akkordfolge über denen längere Achtelkoloraturen ranken. Mit ,laudabo te“ wird der Motettenverlauf erneut impulsiver und komplexer, was die Tastenbearbeitung mit dichter Vollgriffigkeit nachzeichnet. Die virtuosen auf- und abwärtseilenden Skalen bei ,intende“ dienen einer wirkungsvollen Schlussbildung.

Bekannt ist, dass die italienische Orgel der 16. und 17. Jahrhunderts meist ein einmanualiges Prinzipalchorinstrument im Reihenstil war. Wenn Fuß-Pedale vorhanden waren, so waren sie fest an die unteren Tasten des Manuals angehängt. ${ }^{24}$ Die Spiel- und Registriermöglichkeiten dieser Orgel verbinden sich mit dem 1., besonders aber mit dem 2. und 3. Intavolierungstypen, d.h. mit Bearbeitungsformen, bei denen die durch das Vokalstück vorgegebene Satzdynamik nachgezeichnet und durch eine rhythmische und kontrastreiche Spielweise belebt wird. Dies verbindet sich gut mit den Intabulierungsform der Motette Gabrielis, wobei offen bleibt, ob diese Bearbeitung als eigenständiges Stück oder als Begleitstimme angefertigt wurde.

V.

Neben den Intavolierungen von Motetten und Messesätzen kennen wir aus Italien Bearbeitungen von Madrigalen. Zwischen 1593 und 1605 ließ Giovanni Gabrieli Clavierwerke seines Onkels Andrea Gabrieli drucken, die er mit eigenen Stücken ergänzte. Im dritten Band dieser venezianischen Publikationsreihe findet sich „Jo mi son giovinetta“, Madrigale a 4. di Giachet, Tabulato da Andrea Gabrieli. ${ }^{25}$ Tatsächlich komponierte nicht Jaquet de Mantua das kurze Madrigal, ${ }^{26}$ das sich großer Beliebtheit erfreute, sondern Domenico Maria Ferrabosco. ${ }^{27}$ Überliefert ist der Vokalsatz in mehreren Anthologien des 16. und frühen 17. Jahrhunderts. ${ }^{28}$ Verglichen mit der soeben beschriebenen Motettenbearbeitung Giovannis fallen Andrea Gabrielis virtuose Spielfiguren und Skalen auf, welche die Madrigalbearbeitung schematisch durchziehen. Obwohl der Satz vom grifftechnischen Idiom geprägt ist, entdecken wir-viele stimmig konzipierte Passagen. Bei Dominanz der beiden Außenstimmen partizipiert jede Stimme am

24. Vergl. Klotz, S. 132ff.

25. Ediert in A. Gabrieli, Intonationen für Orgel, hrsg. von Pierre Pidoux, Kassel u.a., 1978. S. $32 \mathrm{ff}$.

26. Vergl. Denis Arnold \& Elsie M. Arnold, Art. Gabrieli, Andrea, in: NGroveD, Vol. 7. S. 60.

27. John V. Cockshoot, Art. Domenico Maria Ferrabosco, in: NGroveD, Vol. 6. S. 477.

28. Ediert ist das Madrigal in The Anthologies of Black-Note Madrigals, in: Corpus Mensurabilis Musicae 73, I, Pars 2, ed. Don Harrán, American Institute of Musicology, Stuttgart-Neuhausen, 1978. S. 109ff. 
Passagenwerk. Ein subtiler Umgang mit der Satzdynamik - die Reaktion auf die unterschiedliche Dichte der Klangfelder wie in der oben beschriebenen Motettenintavolierung - gibt es in der Madrigalbearbeitung nicht.

In seinem Primo Libro di Diversi Capricci veröffentlichte Ascanio Mayone, der Hofkapellmeister des spanischen Vizekönigs zu Neapel, eine Intabulierung des Madrigals „Ancidetemi pur“. Jacques Arcadelts schlichtes, kurzes Madrigal stammt aus dem Primo Libro di Madrigali (Venedig, 1539). ${ }^{29}$

Arcadelts Madrigal besteht aus einer überschaubaren Folge einfacher, aber wirkungsvoller musikalischer Einfälle. Sein Aufbau hat die Form A BB' CC'. Die Faktur der Madrigalbearbeitung entspricht Mayones Toccaten aus dieser Sammlung. Daher möchte ich den Begriff Toccateninvolierung verwenden. Mayone lockert das Madrigalschema durch den modifizierten Einsatz unterschiedlich verlaufender Spielfiguren auf. Im Gegensatz zu Andrea Gabrieli vermeidet er schematisch eingesetzte Floskeln. Immer neue, oft sehr virtuose Spielfiguren bringen farbige Vielfalt.

\section{N.B. 3, Beispiele verschiedener Floskeln (Koloraturen) bei Mayone:}

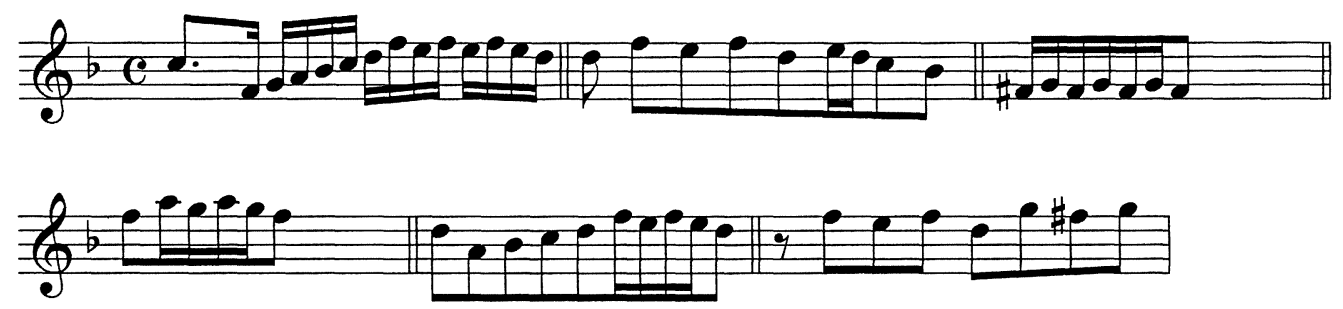

Stufenmelodik, Sprungmelodik, Tonumspielungen und rhythmische Vielfalt prägen die Kolorierung. Im Gegensatz zu den übrigen Toccaten spielen in Mayones Toccateninvolierung Imitationen und Sequenztechnik kaum eine Rolle.

24 Jahre nach Mayone nahm sich der Organista in S. Pietro Di Roma G. Frescobaldi Arcadelts Madrigal an. ${ }^{30}$ Offensichtlich knüpft er an die neapolitanische Tradition des Intavolierens an, die ihrerseits von spanischen Komponisten angeregt sein kann. Außer Mayone veröffentlichte Giovanni Maria Trabaci, ein weiterer Organist aus Neapel, in seinen zwei

29. Ascanio Mayone, Diversi Capricci Per Sonare Libro I Napoli 1603, hrsg. von Christopher Stembridge, Padua, 1981. S. 24-29.

30. Girolamo Frescobaldi, Orgel- und Klavierwerke, Bd. IV, Das zweite Buch der Toccaten, Canzonen usw., hrsg. von Pierre Pidoux, Kassel u.a., 1948. S. 46-51. [= Pidoux, 1948]. 
Clavierbüchern Madrigalbearbeitungen. ${ }^{31}$ Diese zwei Publikationen sowie die beiden Clavierbücher von Mayone erschienen vor Frescobaldis zweitem Toccatenbuch.

$\mathrm{Zu}$ der Madrigalbearbeitung des römischen Komponisten bemerkt Friedrich W. Riedel: „Wäre der Titel des Madrigals nicht beigefügt (der Name des Komponisten fehlt ohnehin), würde man niemals darauf kommen, dass hier ein Vokalstück als Vorlage diente. Der Faktur nach unterschiedet sich die Bearbeitung nicht von den übrigen Toccaten der Sammlung. "32 Die Faktur des Stückes legt es nahe, wie bei Mayone von einer Intavolierungs-Toccata zu sprechen.

Frescobaldis „Ancidetemi pur“ d'Arcadelt passagio ist wie Mayones IntavolierungsToccata von großer, nach vorne drängender Bewegungsdramatik geprägt. Allerdings setzt der römische Komponist die „passagi“ systematischer ein als Mayone in Neapel. Wie der Maestro di cappella aus Neapel gestaltet Frescobaldi mit immer neuen virtuosen Spielfiguren, fließenden Floskeln und Floskelkombinationen die einzelnen Satzabschnitte abwechslungsreich. Anders als der Süditaliener setzt Frescobaldi sein musikalisches Material einmalig ein.

VI.

Als hervorragender Kenner der deutschen Orgelszene seiner Zeit beschreibt Michael Praetorius in der Syntagma Musicum II, De Organographia (Wolfenbüttel, 1619) ${ }^{33} 39$ Dispositionen ,vornehmen OrgelnWerck in Deutschland“. Es handelt sich um nord- und mitteldeutsche Instrumente mit mehreren Werken und eigenständigem Pedalwerk. Mit dieser mehrwerkigen Anlage unterscheiden sich die von Praetorius vorgestellten Orgeln von den italienischen. Daraus resultieren wichtige Unterschiede in der Kompositions- und Intavolierungsart und in der Spielweise dieses Orgeltypus. Da die Pedale und Klaviaturen eigene Werke ansteuern, können Klänge gegeneinander registriert werden. Durch das gleichzeitige Spiel auf mehreren Werken können sich kreuzende Stimmen deutlich hörbar gemacht werden.

Von Scheidemann kennen wir zwölf kunstvolle Intavolierungen von Motetten Orlando di Lassos, Hans Leo Hasslers, Hieronymus Praetorius‘ und Giovanni Bassanos. ${ }^{34}$ Scheidemanns vier Intavolierungsmethoden lassen sich folgendermaßen beschreiben: ${ }^{35}$

„1. Die Werke der einfach strukturierten Tabulatur (...) sind für nur ein Manual und Pedal konzipiert. Die Figuration ist auf die Oberstimme der Motette konzentriert, welche nicht als Solostimme auf einem getrennten Manual bezeichnet ist. Gelegentlich wird die Figuration heruntergelegt, aber der Satz bleibt leicht und kompakt.

31. Apel, S. $432,437 \& 423 \mathrm{ff}$.

32. Riedel, S. 117.

33. Faksimile-Nachdruck, hrsg. von Arno Forchert, Kassel u.a., 2001. S. 161-203.

34. Ediert in: Scheidemann, 12 Orgelintavolierungen, Bd. 1-3, hrsg. von Cleveland Johnson, Wilhelmshaven, 1990/01. [= Johnson] Siehe auch Michael Belotti, Peter Philips and Heinrich Scheidemann or, The Art of Intabulation, in: Proceedings of the Göteborg International Organ Academy, 1994, ed. H. Davidson and S. Jullander, Göteborg, 1995. S. 75-84.

35. Siehe einheitliches Vorwort zu den drei Bänden. 
2. Eine Steigerung bildet die ,doppelt strukturierte Tabulatur“ (Nr. 2 und 5). Diese Werke erhalten zusätzliche Farbe, indem die ausgearbeitete Figuration in der Oberstimme isoliert wird, um einen unabhängigen Vortrag auf einem Solomanual zu erlauben. Die Unterstimmen, die ziemlich genau den originalen Vokalstimmen entsprechend übertragen sind, erfüllen die Aufgabe der Begleitung.

3. Erweitert man den Prozess um einen Schritt, kommt man zur „Phantasie“ (Nr. 1,7 und 12), die im Stil direkt mit der bis in die Bach Zeit florierenden Choralphantasie verbunden ist. Nur dient bei Scheidemann kein Choral, sondern eine Motette als Grundlage. Diese Werke mit ihren schnellen Manualwechseln, zahlreichen Echoeffekten und mehrchörigen Schichtungen waren offensichtlich Schaustücke für die großen Hamburger Orgeln.

4. Die „Manualiter-Tabulatur“ (...) ist Scheidemanns komplexeste Art der Tabulatur, denn diese Werke weisen eine geschlossene, verwobene Struktur auf mit lebhaften Figurationen, die durch alle Stimmen wandern."

Das Gegenüberstellen von kontrastierenden Orgelregistern zeigt sich als weitere Bearbeitungsart der Intavolierungen. Wichtig ist der Hinweis auf die „zusätzlichen Farbe“, welche der figurierten oberen Stimme zusätzliche Qualität gibt und Scheidemanns monodischen Satz charakterisiert. ${ }^{36}$

VI.

Wenden wir uns ausgewählten Bearbeitungen für Tasteninstrumente mehrstimmiger Vokalstücke in anderen Ländern und Regionen zu, so können wir den bei Scheidemann so bemerkenswert auftretenden 5. Kolorierungstypus, dessen Ausführung an eine mehrwerkige (norddeutsche) Orgel mit kontrastierenden Klangfarben und umfangreichen Pedaleinsatz gebunden ist, außer acht lassen.

Zunächst begegnen uns in der Tabulatura Joannis De Lyublyn Canonic[orum] Regularium de Crasnyk. 1540. Motettenbearbeitungen. ${ }^{37}$ Neben Intabulierungen anonymer Vorlagen sind Antoine Brumel, Josquin des Prez, Lupus Hellinck und Claudin Sermisy als Komponisten einiger Motetten bekannt. Die in Johannes von Lublins Tabulatur bearbeitete Motette „Ave Rosa sine spinis“38 wurde von Ludwig Senfl komponiert. ${ }^{39}$ Die Intabulierung ist eine wörtliche Übertragung der Vorlage, praktisch eine mechanische Übernahme ohne jede

36. „Durch die Verwendung von zwei Manualen und Pedal wird ein „monodischer“ Satz möglich: der Diskant erklingt koloriert im Rückpositiv, der Bass wird dem Pedal übertragen, während die Mittelstimmen auf Organo kaum ausgeziert werden und meist nur harmonisch füllende Bedeutung haben." Werner Breig, Die Orgelwerke von Heinrich Scheidemann, Beiheft zum Archiv für Musikwissenschaft 3, Wiesbaden, 1967. S. 97.

37. Ediert in Johannes of Lublin, Tabulature of Keyboard Music, in: Corpus Of Early Keyboard Music 6, Vol. III. Ed. John R. White, Stuttgart-Neuhausen, 1966/R1985.

38. Ebenda, S. 15-21.

39. U.a. ediert in: Das Chorwerk 62, Ludwig Senfl, Zwei Marien=Motetten zu fünf Stimmen, hrsg. von Walter Gerstenberg, Wolfenbüttel, 1957. S. 15-28. 
orgeltypischen Kolorierungstechniken. Die originale Tonhöhe wurde beibehalten. Somit scheinen diese und andere Bearbeitungen aus der polnischen Tabulatur Musik aus didaktischen und archivarischen Gründen kompiliert worden zu sein.

Neben Italien und den deutschsprachigen Landen ist aus Frankreich ein bemerkenswertes, wenn auch kleines Repertoire mit Intabulierungen, besonders von Madrigalen und Chansons bekannt. Die französische Tastenmusik dieser Zeit überliefert zwischen den Messen, Magnificats, Motetten und Liedern, die Pierre Attaingnant 1530/31 veröffentlichte, und den Kirchenhymnen- (1623) und Magnificatversetten (1626) von Jean Titelouze lediglich ein Manuskript mit Intabulierungen eines anonymen Verfassers in französischer Orgeltabulatur. ${ }^{40}$ Die Komponisten der Chansons sind u.a. Pierre Sandrin, Clément Janequin und Nicolas Gombert. Ähnlichkeiten mit den Intabulierungen Attaingnants bringen den anonymen Koloristen mit der französischen Schule in Verbindung. Bonfils beschreibt dies wie folgt:

Es sind bestimmte „barocke“, instrumental konzipierte virtuose Umspielungen, Durchgänge und Ornamente, ${ }^{41}$ beispielsweise in der Bearbeitung der fünfstimmigen Chanson „Vray dieu d'ay mer“ von Descaudin, ${ }^{42}$ die in zeitgleichen italienischen, polnischen, englischen und spanischen Intabulierungen in dieser Konzentration kaum anzutreffen sind.

Deutlich zurückhaltender als die ausgefeilte Kolorierungspraxis des 15. Jahrhunderts Stichwort Buxheimer Orgelbuch - präsentieren sich um 1520 Intabulierungen in Fridolin Sichers Orgeltabulatur. Der Vergleich der vierstimmigen Chanson „Fors seulement“ von Matthaeus Pipelare $^{43}$ mit Sichers Intabulierung im St. Galler Orgelbuch ${ }^{44}$ zeigt in der Tastenfassung den diskreten Einsatz kurzer Durchgänge. Viele lange, aus der vokalen Vorlage wörtlich übernommene Notenwerte am Beginn eines neuen Satzabschnittes und relativ schlichte Kadenzen wurden möglicherweise vom Interpreten durch seine individuelle Diminutionskunst aufbereitet. $\mathrm{Zu}$ diesem Verfahren lädt jedes unkoloriert abgesetzte Stück den phantasievollen Praktiker ein.

VII.

Im England des 16. Jahrhunderts begegnen uns im Mulliner Book neben verschiedenen Orgelstücken Transkriptionen von Anthems. Exemplarisch werfen wir einen Blick auf das

40. Mus. ms 2987 der Bayerischen Staatsbibliothek in München. Ediert in Jean Bonfils, Chansons Françaises pour orgue vers 1550, Paris, 1968. [= Bonfils].

41. Ebenda, S. VII.

42. Ebenda, S. 13ff: Zur Kunst des Intabulierens (in Frankreich) siehe Yvonne Rokseth, La Musique D'Orgue Au XVe. Siègle Et Au Début Du XVIe., Paris, 1930. S. 173ff.

43. Matthaeus Pipelare, Opera Omnia, Corpus Mensurabilis Musicae, Vol. 34, ed. Ronald Cross, American Institute of Musicology, Stuttgart-Neuhausen, 1966. Setting II, S. $11 \mathrm{ff}$.

44. St. Gallen, Codex 530. Ediert ebenda, S. 14 und in Hans Joachim Marx (Hrsg.), St. Galler Orgelbuch. Die Orgeltabulatur des Friedrich Sicher, in Zusammenarbeit mit Thomas Warburton, Basel, 1992. S. 234. 
vierstimmige „Purge me, O Lord“ von Thomas Tallis, das in Mulliners Kompilation unter dem Titel „Fond youth is a bubble“ aufgezeichnet ist. ${ }^{45}$ Bei der „keyboard reduction“, die Thomas Mulliner wohl in den Jahren 1550 bis 1575 anlegte, handelt es sich um eine wörtliche Übernahme des Anthems. Die Tonart beider Fassungen ist identisch. Denkbar ist, dass wie bei Johannes von Lublin (s.o.) mit der Orgelfassung pädagogische und archivarische Intentionen verfolgt wurden - das Aneignen des Satzes als kompositorische Studie. Aber vielleicht diente auch die Orgelfassung des Anthems wie ein Kantionalsatz der Gesangsbegleitung.

Eine Stellung zwischen dem puritanischen Intabulierungsgrad von „Purge me, O Lord“ aus dem Mulliner Book und den ,barocken“ Floskelbewegungen und der energievollen Spielfreude der französischen Stücke aus der Jahrhundertmitte nehmen, ebenfalls anonym überlieferte, handschriftliche Intabulierungen französischer Chansons (u.a. von De Sermisy) und einiger englischer Stücke ein. Diese Bearbeitungen entstanden um $1580 .{ }^{46}$ Reizvoll ist, eine Intabulierung von Pierre Sandrin aus der Münchener Handschrift neben ein koloriertes Stück des gleichen Komponisten aus der Yorker Handschrift zu legen. Die anonyme englische Bearbeitung von „Si mon travel“ überträgt das erste Drittel der Chanson wörtlich für das Tasteninstrument. Im zweiten Drittel lockern einige Passagen und Umspielungen den Notentext auf. In der Schlusszeile verdichtet der anonyme Arrangeur das Passagenwerk quasi als Finalsteigerung. Nur in dieser Schlusszeile nähert sich die englische Intabulierung der Faktur von Sandrins anonymer französischer Bearbeitung ,Je ne puis bonnement“ an, die konsequent koloriert ist. ${ }^{47}$ Der Anfang von „Je ne puis bonnement“ und der Schluss von „Si mon travel“"48 bestätigen dies:

45. Musica Britannica I: The Mulliner Book, ed. Denis Stevens, London, R1954. S. 21/22. Das Anthem ist ediert u.a. in A Tallis Anthologie, 17 Anthems and Motets, compiled and edited by John Milsom, Oxford \& New York, 1992. S. 95-98.

46. U.a. sind solche Intabulierungen in York, Minster Library, MS M 91 überliefert. Musica Britannica LXVI: Tudor Keyboard Music c. 1520-1580. Transcribed and edited by John Caldwell, London, 1995. [= Caldwell] S. XXVII und S. $178 \mathrm{ff}$.

47. Bonfils, S. 35ff.

48. Caldwell, S. 136ff. 
N.B. 4,

a) Chanson ,Je ne puis bonnement“"
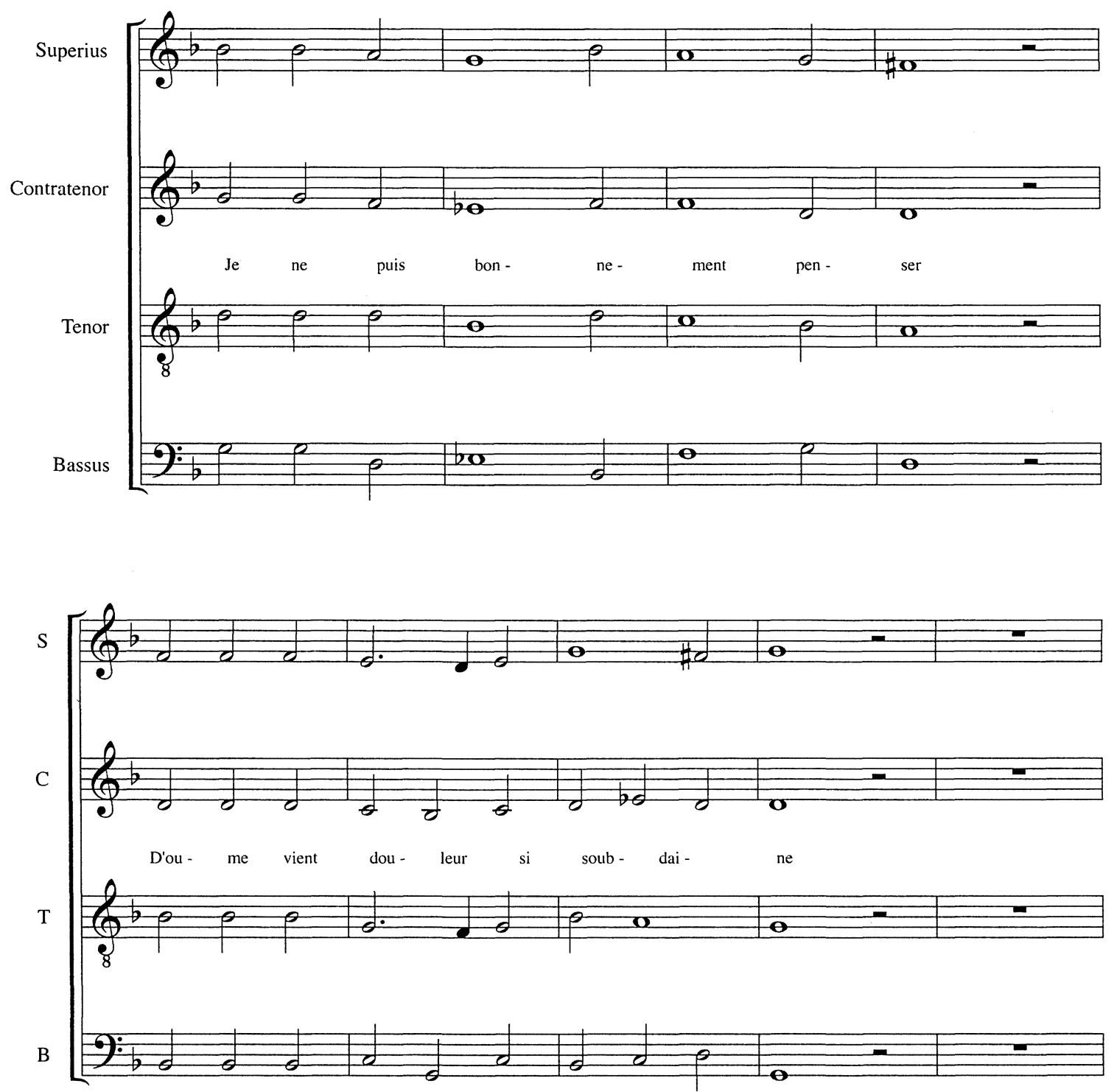
b) Intabulierung „Je ne puis bonnement“:
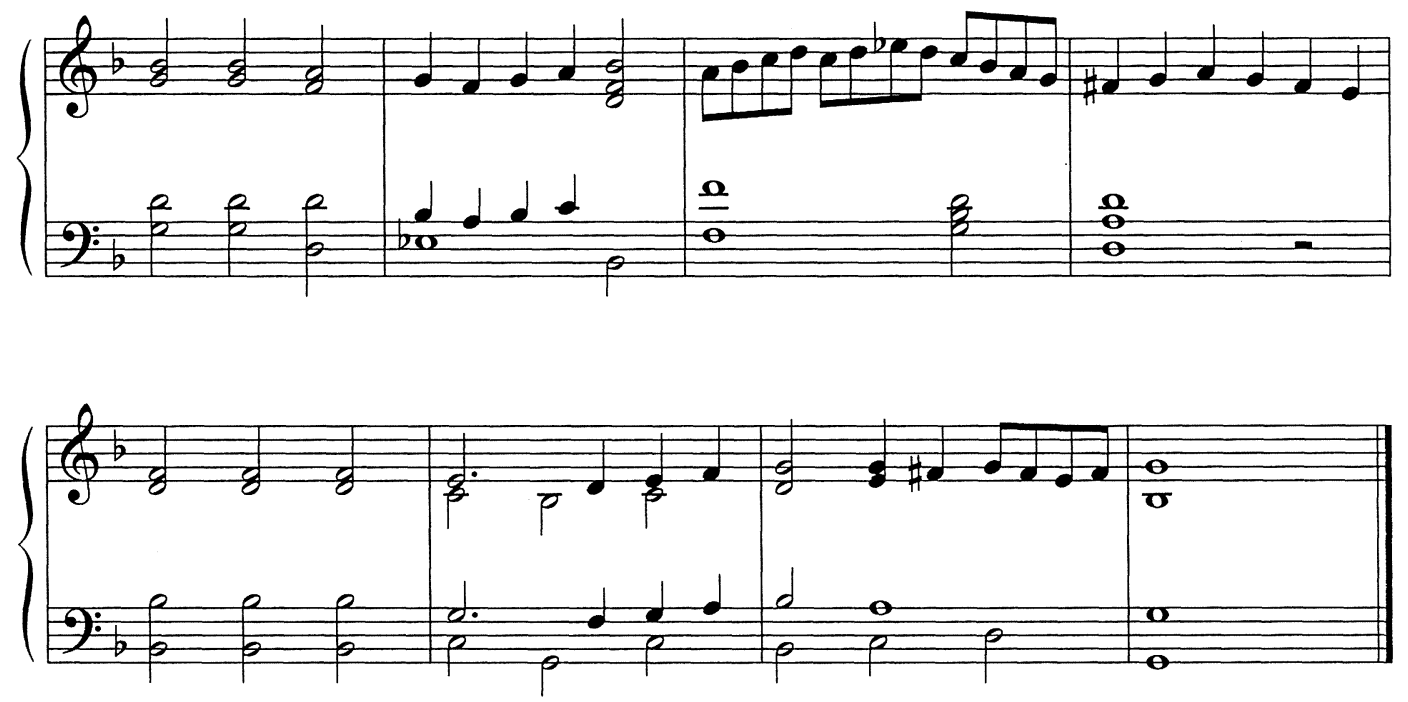

c) Chanson „Si mon travel“:

$\mathrm{S}$

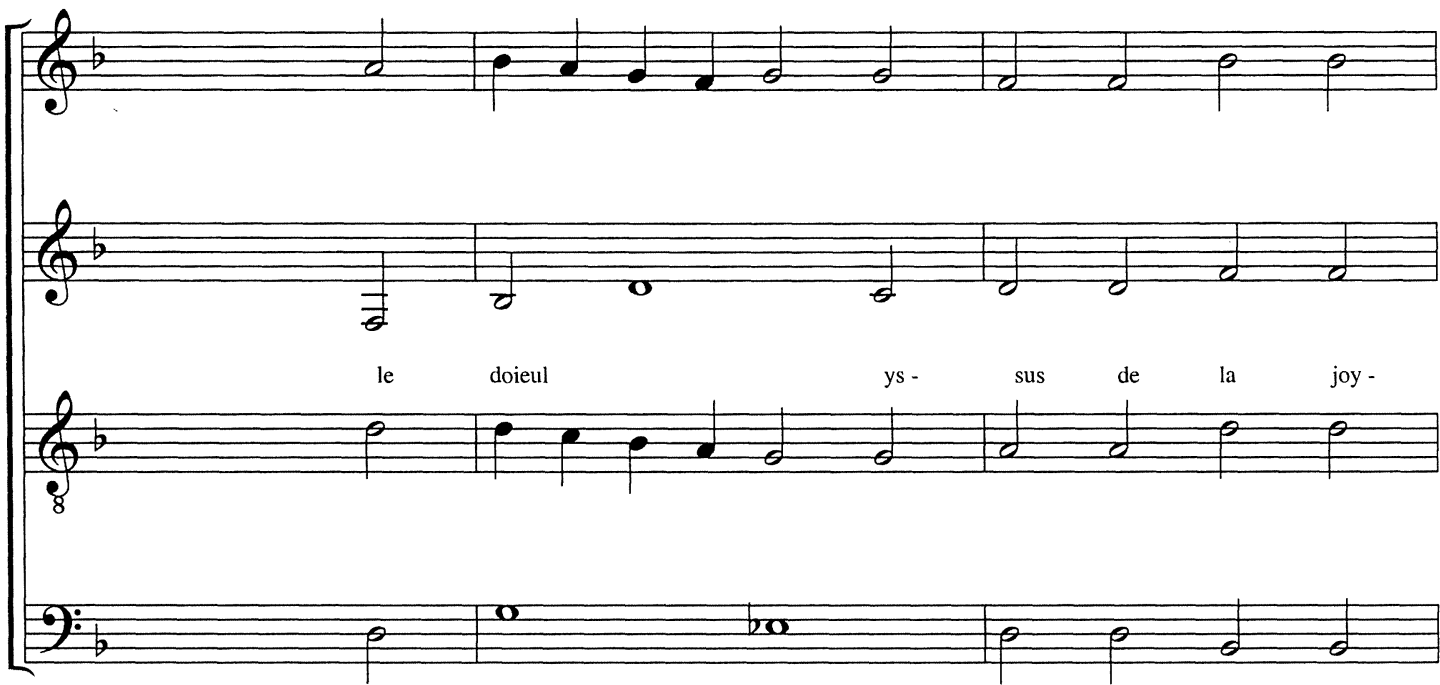


$\mathrm{S}$

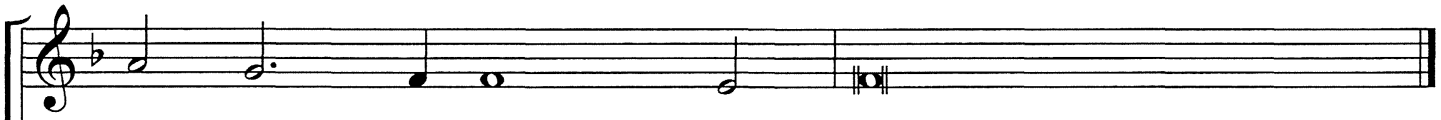

$\mathrm{C}$

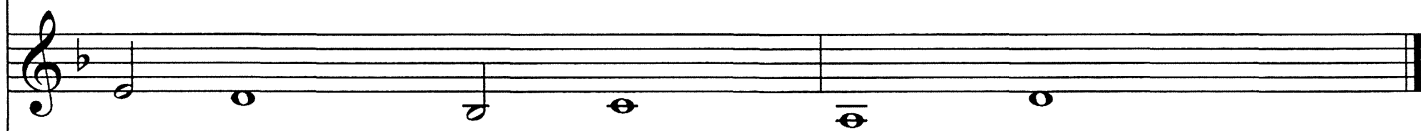

$\mathrm{T}$

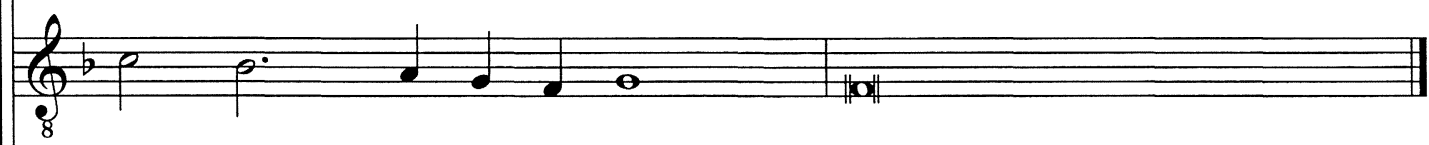

B

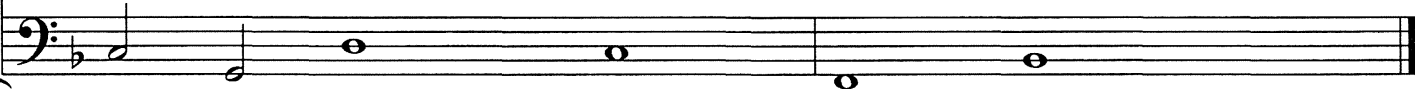

d) Intabulierung „Si mon travel“"
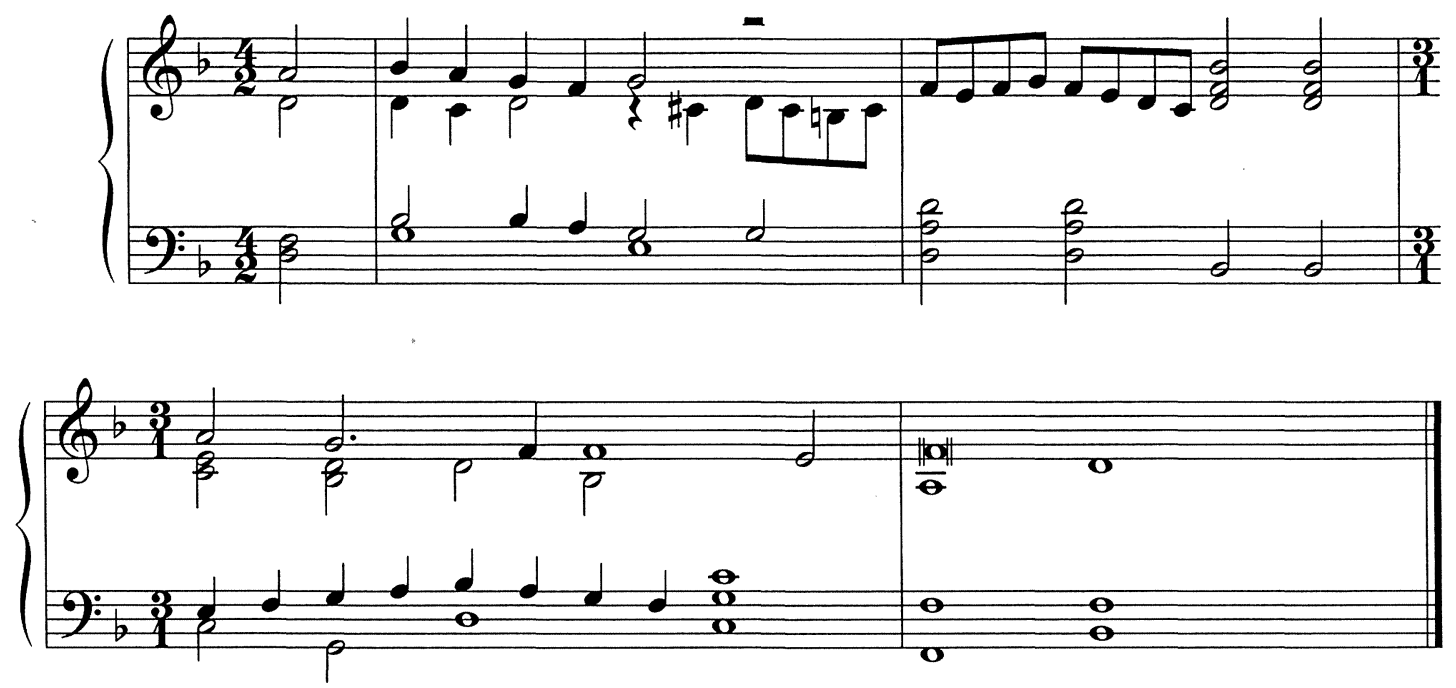

In einer anderen englischen Quelle mit Tastenmusik - das Fitzwilliam Virginal Book fallen besonders Peter Philips' kunstvolle Tastenbearbeitungen vokaler Vorlagen auf. Es waren offensichtlich die modernen und berühmten zeitgenössischen Vokalkompositionen, Chansons Orlando di Lassos und Madrigale Luca Marenzios, Alessandro Striggios und Giulio Caccinis, - die damaligen „Gassenhauer“ - die Philips durch Intabulieren in den musikalischen Alltag eines Palastes und wohlhabenden Bürgerhauses holte. Dies erinnert an die Klavierfassungen (zu 
zwei und vier Händen) u.a. von Stücken aus populären Opern und an die piano arrangements von Pop- und Rocksongs in unseren Tagen.

Besonders bei den Bearbeitungen von Lassos Chansons scheint zuzutreffen, was wir zuvor bei Frescobaldis Toccatenintavolierungen feststellen konnten: Die Faktur der Bearbeitungen von Philips entfernt sich mit ihrem virtuosen und fantasievollen Figurenwerk weit von der vokalen Vorlage. Skelettartig stehen bestimmte Töne und Tonfolgen aus dem Gewebe der Koloraturen hervor, durch welche die Chanson identifiziert werden kann. Ein kurzer vergleichender Blick in Lassos Chanson „Bon Jour mon Coeur“ und Philips“ Tastenarrangement ${ }^{49}$ dokumentiert eine farbige und aufregende Kunst.

N.B. 5,

a) Der Anfang der Chanson von Lasso:

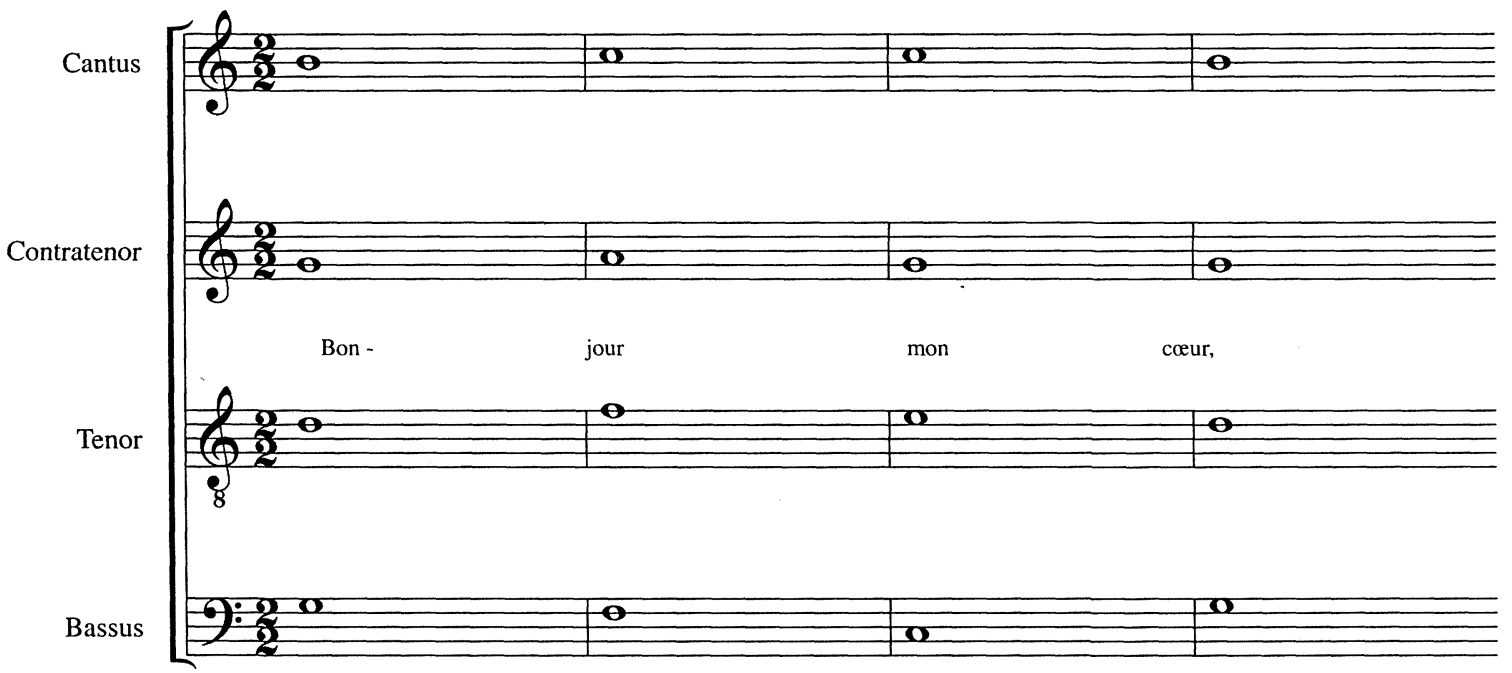

49. Die Chanson ist ediert in Französische Chansons, hrsg. von P. Wehrle und A. Krings, Köln, 1970. S. $26 / 27$. Philips“ Intabulierung ist ediert in The Fitzwilliam Virginal Book, ed. J.A. Maitland and W. Barclay Squire, revised Dover Edition by B. Winogron, New York, 1979-80. S. 317-20. Die Intabulierung ist zusammen mit der Chanson ediert in Musica Britannica LXXV: Peter Philips, Complete Keyboard Music. Transcribed and edited by David J. Smith. London, 1999. S. 815. N.B. 7 ist nach der Edition der Musica Britannica LXXV. 
b) Der Anfang der Intabulierung von Philips:
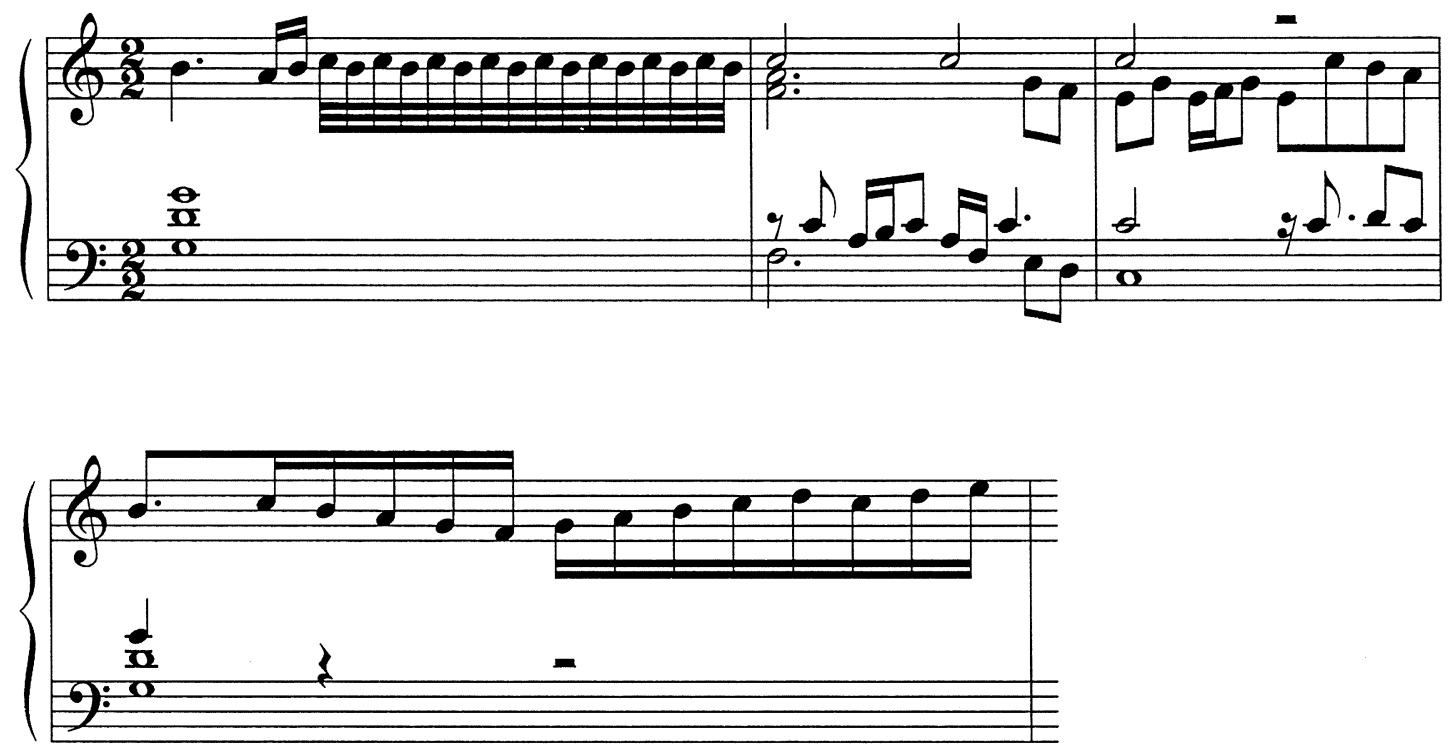

Philips‘ bemerkenswert virtuose Dichte des figürlichen Rankenwerkes ist ansatzweise schon zwei Generationen zuvor in Andrea Gabrielis Bearbeitung der Lasso-Chanson „Susanne un jour" gegeben. ${ }^{50}$ Doch erreicht das Intavolieren, das virtuose Fantasieren, das unbeschwerte Ausleben einer virtuosen Spielfreude bei gleichzeitigem Bezug auf ein vokales Modell seine höchste Kunstfertigkeit besonders bei Philips, zeitgleich in Italien bei Frescobaldi und in den Susanna-Bearbeitungen des Portugiesen Manuel Rodrigues Coelho.

VIII.

Auch aus Spanien sind Bearbeitungen mehrstimmiger Vokalstücke für Tasteninstrumente überliefert. Ein Beispiel ist die „Motete glosado Ave María de Josquin des Prés“ von Antonio de Cabezón. ${ }^{51}$ De Cabezóns Bearbeitung der Motette steht im 2. Ton auf $g$. Mit seiner konsequent linearen Stimmführung übernimmt der spanische Hoforganist die Sechsstimmigkeit der

50. Beide Stücke sind ediert in Kinkeldey, S. 264-74. [= Kinkeldey].

51. Antonio de Cabezón: Claviermusik. Obras de Música para Tecla, Arpa y Vihuela; hrsg. von Macario Santiago Kastner, Mainz u.a., 2/1979. S. 32-37. [= Kastner] Andere Intabulierungen Cabezóns sind ediert in: Thomas Warburton, Keyboard Intabulations of Music by Josquin Des Prez, Recent Researches in the Music of the Renaissance, Vol. XXXIV, Madison, 1980. 
Motettenvorlage. Cabezóns Vorlage ist Josquins secunda pars „Ave Maria“ des sechsstimmigen „Pater noster“. ${ }^{52}$ Die Intavolierung durchwebt den weitgehend homorhythmischen Motettensatz aufwendig mit ausgedehnten skalenartigen Durchgängen. Diese Durchgänge bestehen aus kleinen Notenwerten (Semiminimae). Sie haben den emphatischen andächtigen Charakter der vokalen Vorlage zugunsten eines komplexen instrumentalen Spieles auf.

Cabezóns „Ave Maria“-Intavolierung stellt einen großen Anspruch an die Tastenmusiker. Die besagten Skalenbänder durchziehen alle Stimmen. Vor einigen Binnenkadenzen verdichtet sich das clavieristische Geschehen, wenn in einer Stimme kurzfristig Fusae auftreten. Eine Folge von vier Mensuren mit Dreierrhythmen unterstreicht die Abschnittbildung. Wegen der großen Akkordumfänge kann diese Intavolierung, auch bei Berücksichtigung der kurzen Oktave, nicht mit den Händen alleine bewältigt werden. „Musikalisch gesehen dürften es größere Partien der Bassstimme gewesen sein, die dem Pedal anvertraut waren, was einen Umfang von etwa $\mathrm{C}_{\text {bis }} \mathrm{c}_{-}$ und eine vom Manual unabhängige Registrierung verlangt; in beidem versagten die sonst damals üblichen spanischen Pedale." ${ }^{\text {53 }}$ Denkbar ist, dass der Komponist bei der Ausführung seiner Intavolierung an niederländische Instrumente dachte, die er auf Reisen und durch Beziehungen des spanischen Hofes zu den Niederlanden kennen gelernt hatte. Außerdem ist denkbar, dass das „Ave Maria“ speziell für die Harfe abgesetzt wurde, die auf dem posthum im Druck erschienenen Titelblatt der Werke Cabezóns ${ }^{54}$ erwähnt ist.

Weitere Beispiele für das Umwandeln vokaler Vorlagen liefert Francisco Fernández Palero in der Sammlung Libro de Cifra nueva para tecla, harpa, y vihuela, die Luis Venegas de Henestrosa 1557 anlegte. Palero diminuiert hier das Kyrie I der Missa De Beata Virginis von Josquin..$^{55}$ Ähnlich Cabezóns „Ave Maria“ durchziehen kleine Notenwerte Paleros Klaviersatz. Wie sein Landsmann bedenkt Palero alle Stimmen mit instrumentalen Ausschmückungen, bei leichter Dominanz der oberen Stimme. Diese Glossen verwischen die unterschiedlichen Satzarten der vokalen Vorlage.

Neben Messesätzen Josquins sind im Libro de Cifra nueva Motetten von Jacquet, Philippe Verdelot, Jean Mouton und eine Chanson von Thomas Crécquillon bearbeitet. Einige Intavolierungen dieser Sammlung stammen aus der Feder Cabezóns.

Der bemerkenswert großen Stellenwert, den Motettenintavolierungen auf der iberischen Halbinsel innehatten, wird durch ein portugiesisches Manuskript unterstrichen. Die Handschrift Mus. 43 aus der Biblioteca General der Universität Coimbra enthält u.a. Intavolierungen von Motetten Clemens non Papas, Pierre Cadéacs, Cornelius Canis', Crécquillons, Nicolas Gomberts, Jacques Arcadelts, Adriano Willaerts, Josquins, Verdelots, Claudin De Sermisys, Jean

52. U.a. in der Kapitularbibliothek zu Padua, MS A 17) und im Chorbuch 55 aus den Jahren 1523 und 1534 der Cappella Sistina überliefert. Vergl. Helmut Osthoff, Josquin Desprez, Bd. II, Tutzing, 1965. S. 96ff. Die Motette ist ediert u.a. in A Josquin Anthology, 12 Motets edited by Ross W. Duffin. Introduction by Paul Hillier, Oxford, 1999. S. 54/62-66.

53. Klotz, S. $151 \mathrm{ff}$.

54. Obras de música para tecla, arpa y vihuela ..., Madrid, 1578.

55. Siehe ebenda, S. 30. Ediert in: Monumentos de la música española II, Luys Venegas de Henestrosa, Libro de Cifra Nueva, hrsg. von Higinio Anglés, Barcelona, 1944. S. 146. Die Messe ist ediert u.a. in: Das Chorwerk 42, Josquin Desprez, Missa de Beata Virgine, hrsg. von Friedrich Blume, Wolfenbüttel u.a., 1936. 
Richafords, Jacques Buus', Moutons und Clement Janequins, wobei mit Francisco Soto de Langa, Antonio de Ribera und Cristóbal de Morales auch Vorlagen iberischer Komponisten vertreten sind. ${ }^{56}$

In der ersten Hälfte des 17. Jahrhunderts gibt es auf der iberischen Halbinsel weitere bemerkenswerte Bearbeitungen vokaler Vorlagen von Rodrigues Coelho und Francisco Correa de Arauxo. ${ }^{57}$ Aus späterer Zeit werden keine Intavolierungen überliefert. Als Ausnahme mag eine mutmaßliche Intavolierung aus der Handschrift Huerto ameno de varias flores de música, recogidas de muchos organistas por Fray Antonio Martín von $1709^{58}$ angesehen werden. Die Orgeltranskription „Oh admirabile sacramento“ geht auf ein anonymes Vokalwerk zurück. ${ }^{59}$

Spanische Musiklehrbücher teilen einen Reichtum an Floskeln mit, welche die Musiker beim Komponieren und Absetzen zur Hand nehmen können. Zu nennen sind die Regla para glosar todos los puntos von Diego Ortiz, wobei Ortiz' Verzierungsvorschläge für die Violone auf Tasten- und Saiteninstrumente übertragbar sind, Thomás de Sancta Marías De las glosas para glosar las obras und Cabezóns exemplarische „Faburdones y glosados“60 stellen einen umfangreichen Fundus typisch instrumentaler melodischer Bausteine bzw. Variations-Floskeln zur Verfügung, mit denen mehrstimmige Vokalwerke zu typisch instrumentalen Stücken umgewandelt werden können. Die gleichen Floskeln bzw. Glosas finden wir in originalen Clavierstücken, keineswegs nur auf der iberischen Halbinsel. Diese in spanischen Traktaten kodifizierten Glosas sind musikhandwerkliches Allgemeingut, die uns in Intabulierungen vieler hier erwähnter Komponisten bzw. Arrangeuren begegnen.

\section{Das Erscheinungsbild von Intabulierungen}

Intabulieren bezeichnet sowohl den Prozess der Umwandlung einer mehrstimmigen Vorlage in ein Instrumentalstück als auch dessen Aufzeichnungsweise. ${ }^{61}$ Professionelle Tastenmusiker waren in der Lage, eine mehrstimmige Vorlage ad hoc in ein koloriertes Tastenstück umzuwandeln. Sie waren an eine schriftliche Fixierung nicht gebunden. Dennoch

56. Vergl. Gustave Reese, Music in The Renaissance, New York, 1954. S. 629.

57. Manuel Rodrigues Coelho, Flores De Musica Pera O Instrumento De Tecla, \& Harpa ..., Lissabon, 1620 und: Francisco Correa de Arauxo, Libro de tientos y discursos de música práctica, y theórica de organo intitulado Facultad orgánica, Alcalá, 1626.

58. Biblioteca Nacional, Madrid, Ms. Música 1360.

59. Siehe Spanische Orgelmusik des 17. Jahrhunderts aus „Huerto ameno de varias flores de música“, Bd. II., hrsg. von Sally Fortino, Wien, 1987. Vorwort. Das Stück ist auf den S. 47 u. 48 ediert. Ein Faksimile ist dem Vorwort vorangestellt.

60. Diego Ortiz, Tratado de glosas sobre cláusulas y otros géneros de puntos en la música de violones, Roma, 1553, hrsg. von Max Schneider, Kassel, 1967, hier fol. 20v-24r, Padre Fray Thomas de Sancta María, Libro llamado Arte de tañer Fantasía ..., Valladolid, 1565, hier fol. 58v, 59r\&v; Faksimileedition hrsg. Denis Stevens, Farnborough, 1972 und Hernando de Cabezón, Obras de Música oara Tecla, Arpa y Vihuela ..., Madrid, 1578, hrsg. von Higinio Anglés, Barcelona, 1966. Siehe auch Van Ree Bernard, S. 68-78.

61. Schäfertöns, S. 12, Thurston Dart \& John Morehen, Art. Tablature, in: The New Grove Dictionary of Music \& Musicians, Stanley Sadie (Ed.), Vol. 18. London, 1980. [= NGroveD] S. 506 und Willy Apel, Die Notation der polyphonen Musik, Wiesbaden, 1989. S. 24-59. 
sind schriftlich fixierte hochartifizielle Intabulierungen und Bearbeitungen überliefert. Dabei können Notierungsarten Hinweise auf den spieltechnischen und musiktheoretischen Ausbildungsstand der Tastenmusiker (natürlich auch Lauten-, Vihuela- und Harfenspieler) geben, für welche die Intabulierung aufgezeichnet wurde. Daher sollen an dieser Stelle die Notationsformen der in der vorliegenden Studie genannten Intabulierungs- und Bearbeitungsbeispiele kurz aufgelistet und beschrieben werden. ${ }^{62}$

\begin{tabular}{|c|c|c|}
\hline Klavierpartitur & $\begin{array}{l}\text { Jede Stimme hat ihr eigenes } \\
\text { Notensystem }\end{array}$ & $\begin{array}{l}\text { Bermudo, Mayone und Rodrigues } \\
\text { Coelho }\end{array}$ \\
\hline $\begin{array}{l}\text { Italienische / englische } \\
\text { Orgeltabulatur }\end{array}$ & $\begin{array}{l}\text { Erinnert an unsere heutige } \\
\text { Klavier-notation }\end{array}$ & $\begin{array}{l}\text { Attaingnant, Mulliner Book, Fitz- } \\
\text { william Virginal Book, } \\
\text { Frescobaldi, Philips, Gabrieli, Fray } \\
\text { Antonio Martín }\end{array}$ \\
\hline $\begin{array}{l}\text { Alte deutsche } \\
\text { Orgeltabulatur }\end{array}$ & $\begin{array}{l}\text { Wenigstens eine Stimme ist mit } \\
\text { Buchstaben notiert }\end{array}$ & $\begin{array}{l}\text { Buxheimer Orgelbuch, Buchner, } \\
\text { Lublintabulatur }\end{array}$ \\
\hline $\begin{array}{l}\text { Neue deutsche } \\
\text { Orgeltabulatur }\end{array}$ & Ausschließlich Buchstabennotation & Sicher, Scheidemann \\
\hline $\begin{array}{l}\text { Spanische } \\
\text { Orgeltabulatur }\end{array}$ & $\begin{array}{l}\text { Die Linien sind keine Notenlinien, } \\
\text { sondern entsprechen den (vier) } \\
\text { Stimmen. Zahlen kennzeichnen die } \\
\text { Töne (Zwei Zählweisen }{ }^{63} \text { ) }\end{array}$ & Palero, Cabezón, de Arauxo \\
\hline
\end{tabular}

\section{Postludium:}

Beim Intavolieren ist eine pädagogische Intention meist obsolet. Wir erinnern uns: Hatte doch Frescobaldi den wahren Künstlern das Partiturspiel empfohlen, so setzt Rodrigues Coelho dies stillschweigend voraus. Seine „Flores de Música“ mit den vier Chansonbearbeitungen sind in Partitur gedruckt. Notensätze und Partituren haben als Zielgruppe technisch versierte Instrumentalmusiker ohne große musiktheoretischen Kenntnisse. ${ }^{64}$

Ein wichtiges Kriterium beim Intabulieren vokaler Vorlagen war für die Arrangeure die Erhaltung der zeitlich-räumlichen Dimension. Gemeint ist damit: die lineare metrische Substanz

62. Ein Großteil der Notationsformen findet man als Faksimiles in Apel, 1970 oder in den entsprechenden Neuausgaben.

63. Apel, 1970. S. $52 \mathrm{ff}$.

64. $\mathrm{Zu}$ den Unterschieden zwischen den hauptsächlich handwerklich-spieltechnisch ausgerichteten Arbeiten der deutschen Koloristen des späten 15. Jahrhunderts und den (zusätzlich) musiktheoretisch fundierten Bearbeitungen vokaler Vorlagen, vornehmlich aus Italien und Spanien (beginnend mit dem frühen 16. Jahrhundert) siehe Kinkeldey, S. 84, 95 und 122. 
der vokalen Vorlagen wird in das Tastenarrangement übernommen. Dies trifft in erster Linie auf die nicht glossierten Stücke zu. In anderen Fällen, z.B. den Stücken von Rodrigues Coelho, ist die vokale Vorlage ziemlich frei bearbeitet: Hier ist die Gattungsgrenze der eigentlichen Intavolierung übertreten.

Das Instrument kann ein weiterer Faktor sein, der den Grad der Intabulierung bestimmt. Gerade Scheidemann zeigt, dass er die Möglichkeiten der norddeutschen, mehrwerkigen Orgel mit (eigenständigem Pedal) beim Intavolieren berücksichtigt. Bei anderen Intavolierungen, hier denke ich besonders an Philips, weißt das Passagenwerk auf ein Kielinstrument hin.

Doch darf nicht vergessen werden, dass auch Bearbeitungen vokaler Stücke für andere Instrumente existieren. Als prominentes Beispiel wurde der Tratado de glosas ... von Ortiz erwähnt. Einige von Scheidemanns Motettenintabulierungen nutzen das mehrwerkige Angebot der norddeutschen Orgel aus, wenn der Komponist die Registriermöglichkeiten konträrer Stimmcharaktere auslotet. Scheidemanns Motettenbearbeitungen markieren einen letzten Höhepunkt und den Schlusspunkt einer Claviermusikgattung. Anders als frühere Motetten- und Messsatzintabulierungen dienten sie als Schaustücke für die großen Hamburger Orgeln. ${ }^{65}$ Verdrängt wurden die Intabulierungen im kirchlichen Raum von dem anwachsenden Bestand genuiner Orgelmusik. Intabulierungen wurden einfach nicht mehr gebraucht.

Bei den englischen und italienischen Orgeln können wir meist von einem einmanualigen Instrument ohne eigenständiges Pedalwerk ausgehen. Genau in diesen Ländern tendieren die Tastenbearbeitungen sakraler vokaler Vorlagen zur wörtlichen Umsetzung oder zumindest zu einem diskreten Bearbeitungsgrad. Anders verhält es sich mit den Bearbeitungen profaner Vorlagen, die ganz den Geschmack der Zeit widerspiegeln. Deren virtuose Ergebnisse können wir bei A. Gabrieli, Frescobaldi, Mayone, Philips und dem anonymen französischen Meister bewundern. Womöglich wurden die (nahezu) wörtlichen Arrangements vokaler Vorlagen von den Musikern ad hoc individuell mit Glossen ausgeschmückt.

Nationale Schulen des Intabulierens lassen sich bei der exemplarischen Beschäftigung mit diesem Thema nicht erkennen. Innerhalb einer Region und eines überschaubaren Zeitraumes können Intabulierungen für Tasteninstrumente stark voneinander abweichen. Doch sind Bearbeitungen mehrstimmiger Vorlagen von dem jeweiligen Tasteninstrument, z.B. der jeweiligen Orgellandschaft, abhängig. Hinzu kommt, dass sich gegen Ende des 16. Jahrhunderts eine differenzierte Auffassung bezüglich der Tasteninstrumente entwickelte. Der Hinweis in bestimmten Publikationen da cantare \& sonare d'ogni sorte de Strumenti, den wir z.B. 1553 im Titel zu Francesco Bendusis Opera nova de balli (Gardano, Venedig) lesen können, erfährt 1576 durch Antonio Valentes Intavolatura de Cimbalo eine eindeutige Zuweisung auf die besaiteten Tasteninstrumente. ${ }^{66}$ Wenn in Frescobaldis Toccate D'Intavolatura Di Cimbalo Et Organo ...

65. Johnson, Vorwort, S. 3.

66. In diesem Zusammenhang ist auch auf die Intavolature Nova di varie sorte de Balli da sonare per arpichordi, Clavicembali, Spinette \& Manachordi, Gardano, Venedig, 1551 hinzuweisen. Vergleiche: The Keyboard Repertoire 18, Antonio Valente, Ricercars, Chansons and Danses from Intavolatura de Cimbalo 1576, ed. Bernard Thomas, London, 1981. Vorwort. Bendusi ist ediert in: Der Bläserchor, Heft 2, hrsg. von Helmut Mönkemeyer, Celle, 1965. 
Libro Primo (Rom, 1637) ${ }^{67}$ Cembalisten und Organisten angesprochen sind, so hat dies ökonomische Gründe; eine größere Käufergruppe sollte angesprochen werden.

Oft kennen wir den konkreten Anlass zur Intavolierung eines Musikstückes nicht. M. Praetorius formuliert einen pädagogischen Ansatz, d.h. der Musiker soll sich durch die Technik des Intabulierens eine Komposition aneignen. Durch das schriftlich fixierte Studienmaterial konnten sich die Musiker und die Zuhörer mit der vokalen Vorlage vertraut machen.

Die anonyme Überlieferung vieler mitunter sehr kunstvoll gestalteten Stücke in Frankreich und England verweist auf einen handwerklichen und spielpraktischen Ansatz. Solche Bearbeitungen hatten hier nicht den Stellenwert einer individuellen Kompositionskunst. Das Intavolieren konnte kunstfertig von den Meistern aus dem Stehgreif bewerkstelligt werden.

Ein nächster Aspekt mag sein, dass eine Orgelfassung einer Motette, ich denke dabei an Gabrielis „Exaudi Domine“, als Begleitfassung - als ausformulierte Orgelstimme - bei der vokalen Ausführung der Motette Verwendung fand. Außerdem wurden Intabulierungen liturgisch verwendet.

Die hier vorgelegte Übersicht kann die Kunst des Intabulierens nur ansatzweise thematisieren. Konkrete Aussagen lassen sich nur durch genaue Analysen und weitergehende stilkritische Untersuchungen gewinnen. Dazu möchte diese Arbeit anregen.

67. Girolamo Frescobaldi, Orgel- und Klavierwerke, Band III. Das erste Buch der Toccaten, Partiten usw. 1637, Kassel u.a., 1954. 